\title{
Simultaneous Determination of Nine Active Compounds of the Traditional Chinese Medicinal Prescription Shaoyao-Gancao-Tang and Analysis of the Relationship between Therapeutical Effect and Compatibility of Medicines
}

\author{
Guangwei Zhu, ${ }^{1}$ Guijun Zhang, ${ }^{1}$ Meng Wang, ${ }^{1}$ Jingjuan Wang, \\ Weixin Zeng, ${ }^{2}$ and Xiaomei Gao ${ }^{1}$ \\ ${ }^{1}$ School of Traditional Chinese Materia Medica, Beijing University of Traditional Chinese Medicine, Beijing 100102, China \\ ${ }^{2}$ Department of Pharmacy, Beijing Shijitan Hospital, Beijing 100038, China
}

Correspondence should be addressed to Guijun Zhang; guijunzhang@163.com

Received 28 June 2014; Revised 14 August 2014; Accepted 28 August 2014; Published 6 November 2014

Academic Editor: Seung-Heon Hong

Copyright (C) 2014 Guangwei Zhu et al. This is an open access article distributed under the Creative Commons Attribution License, which permits unrestricted use, distribution, and reproduction in any medium, provided the original work is properly cited.

\begin{abstract}
A simple and sensitive HPLC-DAD detection method was established for the simultaneous determination of nine compounds including oxypaeoniflorin, albiflorin, paeoniflorin, benzoylpaeoniflorin, glycyrrhizic acid, liquiritin, isoliquiritin, liquiritigenin, and isoliquiritigenin in the Traditional Chinese Medicinal Prescription Shaoyao-Gancao-Tang (SGT) and we analyze the relationship between therapeutical effect and compatibility of medicines by using an Agilent extend-C18 column at a flow rate of $1 \mathrm{~mL} / \mathrm{min}$. The column temperature was maintained at $30^{\circ} \mathrm{C}$ and the detection wavelength was set at $230 \mathrm{~nm}$ for oxypaeoniflorin, albiflorin, paeoniflorin, benzoylpaeoniflorin, and glycyrrhizic acid; $276 \mathrm{~nm}$ for liquiritin and liquiritigenin; $360 \mathrm{~nm}$ for isoliquiritin and isoliquiritigenin. The total contents of the nine compounds in SGT varied from 4.65 to $20.06 \mathrm{mg} / \mathrm{mL}$. The results of this study showed that the content of chemical compounds of Traditional Chinese Medicinal Prescription is mainly influenced by the dosage and compatibility of medicines and the therapeutical effect of Traditional Chinese Medicinal prescription is mainly influenced by the dosage and compatibility of medicines. The method could be suitable for quality control of SGT with bioactive multicompounds.
\end{abstract}

\section{Introduction}

SGT is a Traditional Chinese Prescription containing Radix Paeoniae alba (stir-baked with vinegar) and Radix Glycyrrhizae (stir-baked with honey) with the ratio of $1: 1$ and is commonly used to relieve spasm and pain. It was originally described in Treatise on Febrile Diseases, a medical classic written by Zhongjing Zhang in the 3rd century.

SGT contains many chemical constituents. Oxypaeoniflorin, albiflorin, paeoniflorin, liquiritin, isoliquiritin, liquiritigenin, benzoylpaeoniflorin, isoliquiritigenin, and glycyrrhizic acid (Figure 1) are the most important bioactive constituents reported at present. Albiflorin has the effects in the prevention of osteoporosis and inhibitory effects on DNA cleavage $[1,2]$. Paeoniflorin has been widely investigated as an anti-inflammatory, cognitive enhancer, endotheliumdependent vasodilator, and neuroprotective agent [3-7]. Liquiritigenin and glycyrrhizic acid inhibit liver cell injury and were given intravenously for the treatment of chronic viral hepatitis and cirrhosis in Japan [8,9]. It has also proven itself effective in the treatment of autoimmune hepatitis in one clinical trial [10]. These effects are related to the inhibition of cortisol metabolism within the kidney [11]. Other side effects include [12] headache, transient visual loss, tachycardia, cardiac arrest, hypokalaemia, acute kidney, muscle weakness, myopathy, myoglobinuria, rhabdomyolysis, and increased body weight. Isoliquiritigenin is a chalcone compound and has valuable pharmacological properties such as antioxidant, anti-inflammatory, anticancer, and antiallergic activities. Isoliquiritin and liquiritin have the 


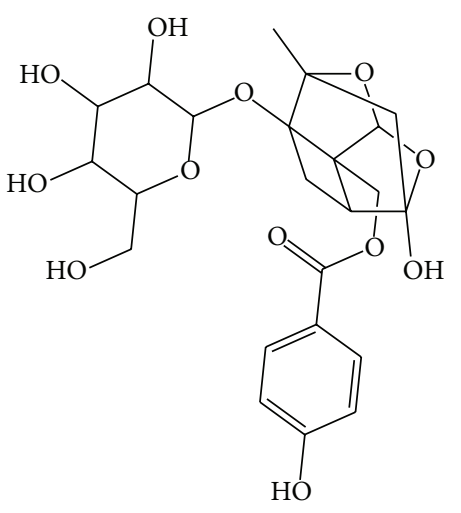

Oxypaeoniflorin

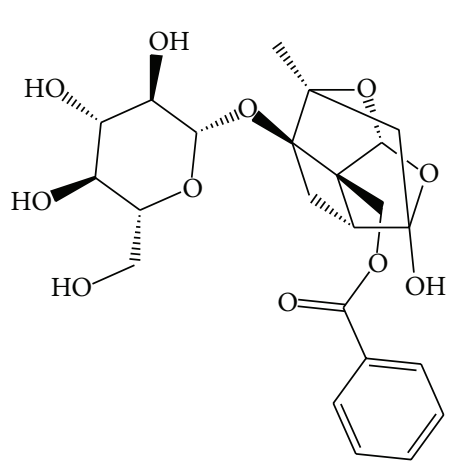

Paeoniflorin<smiles>O=C1C[C@H](c2ccc(O[C@@H]3O[C@H](CO)[C@@H](O)[C@H](O)[C@H]3O)cc2)Oc2cc(O)ccc21</smiles>

Liquiritin<smiles></smiles><smiles>C[C@]12C[C@H](COC(=O)c3ccccc3)[C@@](O)(C(=O)O1)[C@]2(O)O[C@@H]1O[C@H](CO)[C@@H](O)[C@H](O)[C@H]1O</smiles>

Albiflorin<smiles>O=C(/C=C/c1ccc(O[C@@H]2O[C@H](CO)[C@@H](O)[C@H](O)[C@H]2O[C@@H]2OC[C@](O)(CO)[C@H]2O)cc1)c1ccc(O)cc1O</smiles>

Isoliquiritin<smiles>O=C1C[C@H](c2ccc(O)cc2)Oc2cc(O)ccc21</smiles>

Liquiritigenin<smiles>O=C(/C=C/c1ccc(O)cc1)c1ccc(O)cc1O</smiles>

(a)

Figure 1: Continued. 


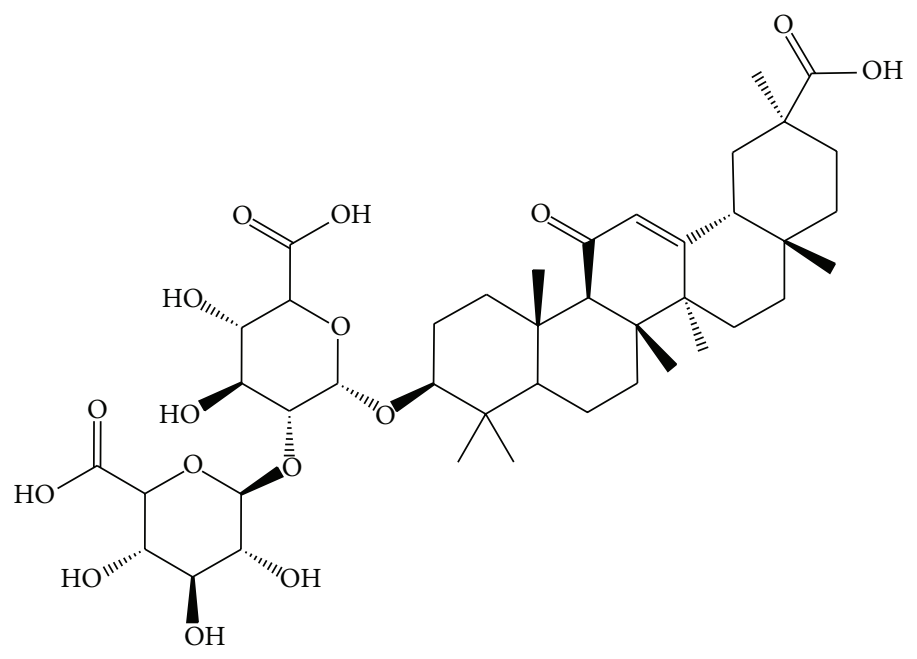

Glycyrrhizic acid

(b)

FIGURE 1: The structures of nine constituents in SGT.

pharmacological activities of antiulcer [13], antioxidative [14], antimicrobial [15], and antitumor [16] activities and others. Oxypaeoniflorin and benzoylpaeoniflorin have the activities of anti-inflammatory, antianxiety, analgesia, spasmolysis, and anticonvulsant [17]. When the nine compounds are mixed in different proportions, they also have different effects. Traditionally, SGT is used clinically for its antispasmodic and muscle relaxation effects in the treatment of leg cramps, stomachache, and menstrual colic pain [18-21]. In the later days, SGT changed, and also its clinical efficacy changed (Table 1).

Modern research has shown that SGT has the remarkable curative effect to the many kinds of diseases. Recently, other pharmacological properties of SGT, such as antiinflammatory, antioxidative, and neuroprotective effects, have also been reported $[22,23]$. In Japan, SGT is one of the predominant traditional Chinese formulations clinically used and is widely applied for the treatment of abdominal pain [24], sometimes together with analgesics and antispasmodics such as anticholinergic drug [25]. As SGT is also used to promote the healing of peptic ulcer, it may also combine with synthetic antisecretory drugs as a histamine $\mathrm{H}_{2}$-receptor antagonist and triple therapy using OAM, a combination of a proton pump inhibitor to reduce gastric acid secretion and two antibiotics to remove Helicobacter pylori [26].

However, very few reports on the quality control of SGT have been published so far. Most methods for quality control of TCM only analyze monomeric compound, which is insufficient as they do not reveal all the compounds present in the chromatographic profile [27]. These studies are not in accord with Traditional Chinese Medical (TCM) theory. The theory suggests that the efficacy of TCM is decided by many different kinds of chemical constituents with bioactivities (Figure 2). Basically, these chemicals are similar to TCM in terms of treatment effect.
Studies have shown that a high percentage of relevant studies on traditional Chinese medicine are in Chinese databases. Fifty percent of systematic reviews on TCM did not search Chinese databases, which could lead to a bias in the results [28]. Many systematic reviews of TCM interventions published in Chinese journals are incomplete, some contained errors or were misleading [29]. In this study, we focused on establishing an effective method to evaluate the quality of SGT for its safe use in clinical practice. In the present study, a HPLC-DAD method was first developed to analyze multicompounds including oxypaeoniflorin, albiflorin, paeoniflorin, liquiritin, isoliquiritin, liquiritigenin, benzoylpaeoniflorin, isoliquiritigenin, and glycyrrhizic acid for quality control of SGT.

Figure 2 illustrates two research methods of traditional Chinese Medicine. The former focus on monomeric compound and the latter focus on multicompounds. TCM is a complex system, which consists of many kinds of herbs. Each herb performs one or more specific functions when used alone. However, they will play a role in the treatment of more when used in combination. Why? The answer is that TCM contains many chemical components, they influence each other (sometimes produce one or more new substances) and result in different therapeutical effects. These effects were caused not by a monomeric compound, but by multicompounds. So, the later method should be the best choice.

\section{Materials and Methods}

2.1. Chemicals and Reagents. The reference compounds of oxypaeoniflorin, albiflorin, paeoniflorin, liquiritin, isoliquiritin, liquiritigenin, benzoylpaeoniflorin, isoliquiritigenin, and glycyrrhizic acid (purity $\geq 98 \%$ ) were purchased from Chengdu Pufeide Biotech Co., Ltd. of China (Chengdu, China). Medicinal materials were purchased from Hebei anguo Chinese 


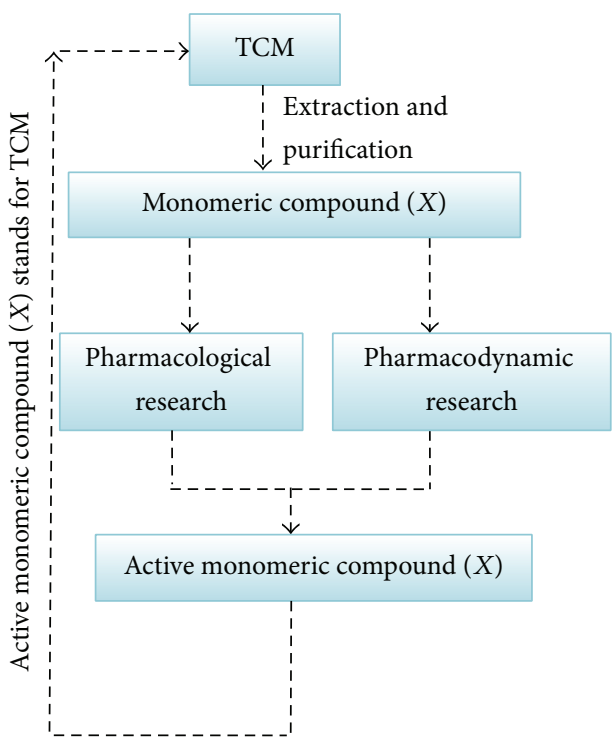

(a) Analyze only monomeric compound

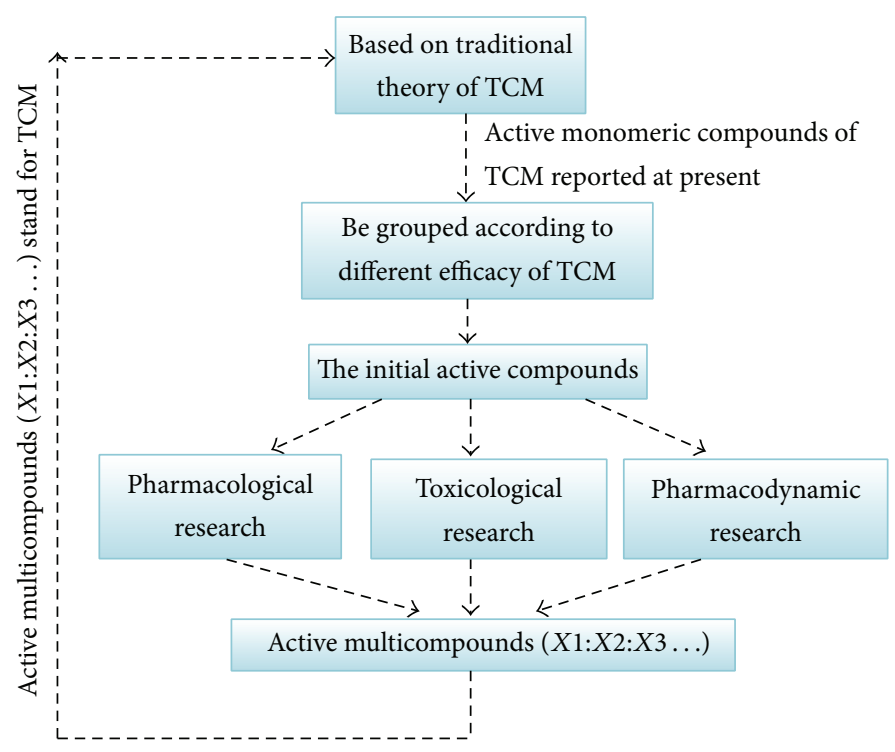

(b) Analyze multicompounds

Figure 2: Studies on TCM.

TABLE 1: Compatibility of medicines, ratio, and therapeutical effect of SGT in different period.

\begin{tabular}{|c|c|c|c|c|}
\hline Reference & Period & Compatibility of medicines & $\begin{array}{l}\text { Ratio } \\
(w / w)\end{array}$ & Therapeutical effect \\
\hline Shanghanlun & In the Han dynasty & $\begin{array}{l}\text { Radix Paeoniae alba (Stir-baked } \\
\text { with vinegar) } \\
\text { and Radix Glycyrrhizae } \\
\text { (stir-baked with honey) }\end{array}$ & $1: 1$ & $\begin{array}{l}\text { For relieving pain in the } \\
\text { legs }\end{array}$ \\
\hline Chuanxinshiyongfang & In the Song dynasty & $\begin{array}{l}\text { Radix Paeoniae Rubra (raw) } \\
\text { and Radix Glycyrrhizae } \\
\text { (stir-baked with honey) }\end{array}$ & $12: 1$ & $\begin{array}{l}\text { For relieving swelling and } \\
\text { pain in the legs }\end{array}$ \\
\hline Zhushijiyanfang & In the Song dynasty & $\begin{array}{l}\text { Radix Paeoniae alba (raw) } \\
\text { and Radix Glycyrrhizae (raw) }\end{array}$ & $3: 2$ & $\begin{array}{l}\text { For relieving pain caused } \\
\text { by diabetes }\end{array}$ \\
\hline Zhushijiyanfang & In the Song dynasty & $\begin{array}{l}\text { Radix Paeoniae Rubra (raw) } \\
\text { and Radix Glycyrrhizae (raw) }\end{array}$ & $6: 1$ & For foot weakness \\
\hline Weishijiacangfang & In the Song dynasty & $\begin{array}{l}\text { Radix Paeoniae alba (raw) } \\
\text { and Radix Glycyrrhizae } \\
\text { (stir-baked with honey) }\end{array}$ & $12: 1$ & For wet beriberi \\
\hline Yimenbafa & In the Qing dynasty & $\begin{array}{l}\text { Radix Paeoniae alba (raw) } \\
\text { and Radix Glycyrrhizae (raw) }\end{array}$ & $3: 1$ & For peratodynia \\
\hline
\end{tabular}

Herbal Medicine Co., Ltd. of China (Hebei, China). Acetic acid was purchased from Tianjin Chemical Regent Co., Ltd. (Tianjin, China), methanol and acetonitrile were HPLCgrade (Fisher, USA) and high purity water was obtained from Wahaha Co., Ltd. (Hangzhou, China).

2.2. Instrumentation and Separation Conditions. Liquid chromatographic analysis was performed on an Agilent HPLCDAD system, which was equipped with an Agilent 1260 infinity DAD (DEAA306741), an Agilent 1260 infinity autosampler (DEAAC23211), an Agilent 1260 infinity column heater (DEACN25004), an Agilent 1260 infinity pump (DEAB709020), and an Agilent extend-C18 (4.6 mm $\times$ $250 \mathrm{~mm}, 5 \mu \mathrm{m}$ ) column. The binary gradient elution system consisted of solvent A (acetonitrile) and solvent B ( $0.1 \%$ acetic acid water). Optimum separation was achieved by using the gradient program described in Table 2 . The column temperature was maintained at $30^{\circ} \mathrm{C}$. The autosampler was conditioned at $25^{\circ} \mathrm{C}$ and injection volume was $10 \mu \mathrm{L}$. The flow rate was $1 \mathrm{~mL} / \mathrm{min}$. The raw data was detected by an HPLCDAD (Agilent1260, USA) and the wavelengths were shown in Table 3.

2.3. Sample Preparation. Medicinal materials (Table 4) were immersed in distilled water $(1: 10, \mathrm{w} / \mathrm{v})$ and boiled twice for $30 \mathrm{~min}$ at each time. The filtrates from each decoction were mixed and concentrated to $1 / 2$ of its original volume; the concentrate solution was thoroughly mixed 
TABLE 2: The binary gradient elution system.

\begin{tabular}{|c|c|c|c|c|c|c|c|c|c|}
\hline Time (min) & $0 \sim 5$ & $5 \sim 12$ & $12 \sim 15$ & $15 \sim 20$ & $20 \sim 25$ & $25 \sim 30$ & $30 \sim 40$ & $40 \sim 50$ & $50 \sim 60$ \\
\hline A: acetonitrile (\%) & $5 \sim 10$ & $10 \sim 14$ & $14 \sim 16$ & $16 \sim 18$ & $18 \sim 20$ & $20 \sim 22$ & $22 \sim 25$ & $25 \sim 40$ & $40 \sim 55$ \\
\hline
\end{tabular}

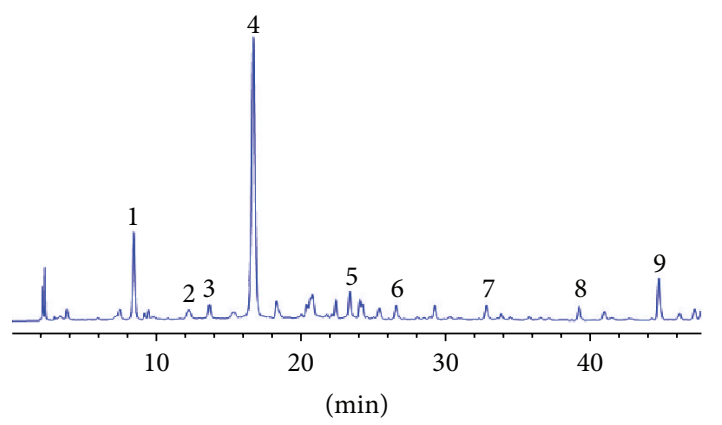

(a)

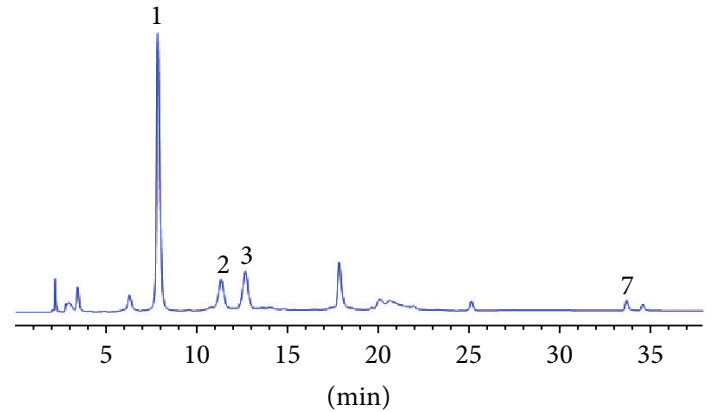
(1) Oxypaeoniorin
(2) Albiorin
(3) Paeoniorin
(4) Liquiritin
(5) Isoliquiritin

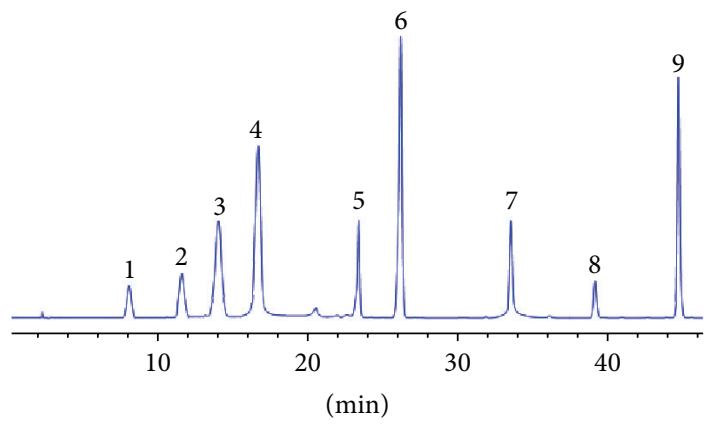

(b)

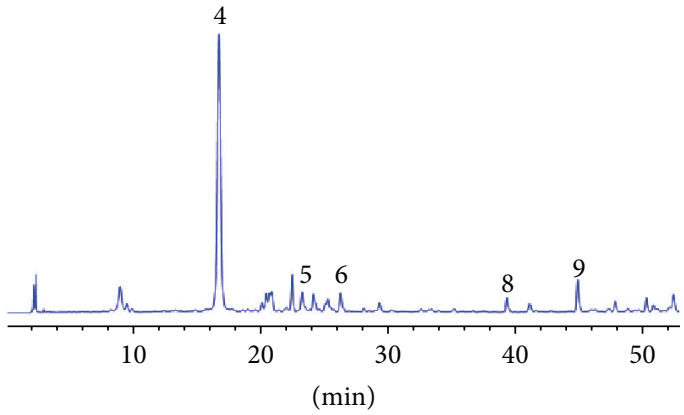
(6) Liquiritigenin
(7) Benzoylpaeoniorin
(8) Isoliquiritigenin
(1) Oxypaeoniorin
(2) Albiorin
(3) Paeoniorin
(4) Liquiritin
(5) Isoliquiritin

(6) Liquiritigenin

(7) Benzoylpaeoniorin

(8) Isoliquiritigenin

(9) Glycyrrhizic acid

(d)

FIGURE 3: HPLC chromatograms of SGT test sample (a), standard mixture (b), test sample without Radix Glycyrrhizae (stir-baked with honey) (c), and test sample without Radix Paeoniae alba (Stir-baked with vinegar) (d).

TABLE 3: Wavelengths.

\begin{tabular}{lccc}
\hline Wavelengths $(\mathrm{nm})$ & 230 & 276 & 360 \\
\hline & Oxypaeoniflorin & & \\
albiflorin, & paeoniflorin, \\
Compounds & genzoylpaeoniflorin, and & Liquiritin and & Isoliquiritin and \\
& glycyrrhizic acid & liquiritigenin & \\
\hline
\end{tabular}

TABLE 4: Compatibility of medicines and dosage of SGT.

\begin{tabular}{lc}
\hline Compatibility of medicines & Dosage \\
\hline Radix Paeoniae alba (stir-baked with vinegar) and Radix Glycyrrhizae (stir-baked with honey) & $12 \mathrm{~g}$ and $12 \mathrm{~g}$ \\
Radix Paeoniae Rubra (raw) and Radix Glycyrrhizae (stir-baked with honey) & $12 \mathrm{~g}$ and $1 \mathrm{~g}$ \\
Radix Paeoniae alba (raw) and Radix Glycyrrhizae (raw) & $12 \mathrm{~g}$ and $8 \mathrm{~g}$ \\
Radix Paeoniae Rubra (raw) and Radix Glycyrrhizae (raw) & $12 \mathrm{~g}$ and $2 \mathrm{~g}$ \\
Radix Paeoniae alba (raw) and Radix Glycyrrhizae (stir-baked with honey) & $12 \mathrm{~g}$ and $1 \mathrm{~g}$ \\
Radix Paeoniae alba (raw) and Radix Glycyrrhizae (raw) & $1400 \mathrm{~mL}$ \\
\hline
\end{tabular}




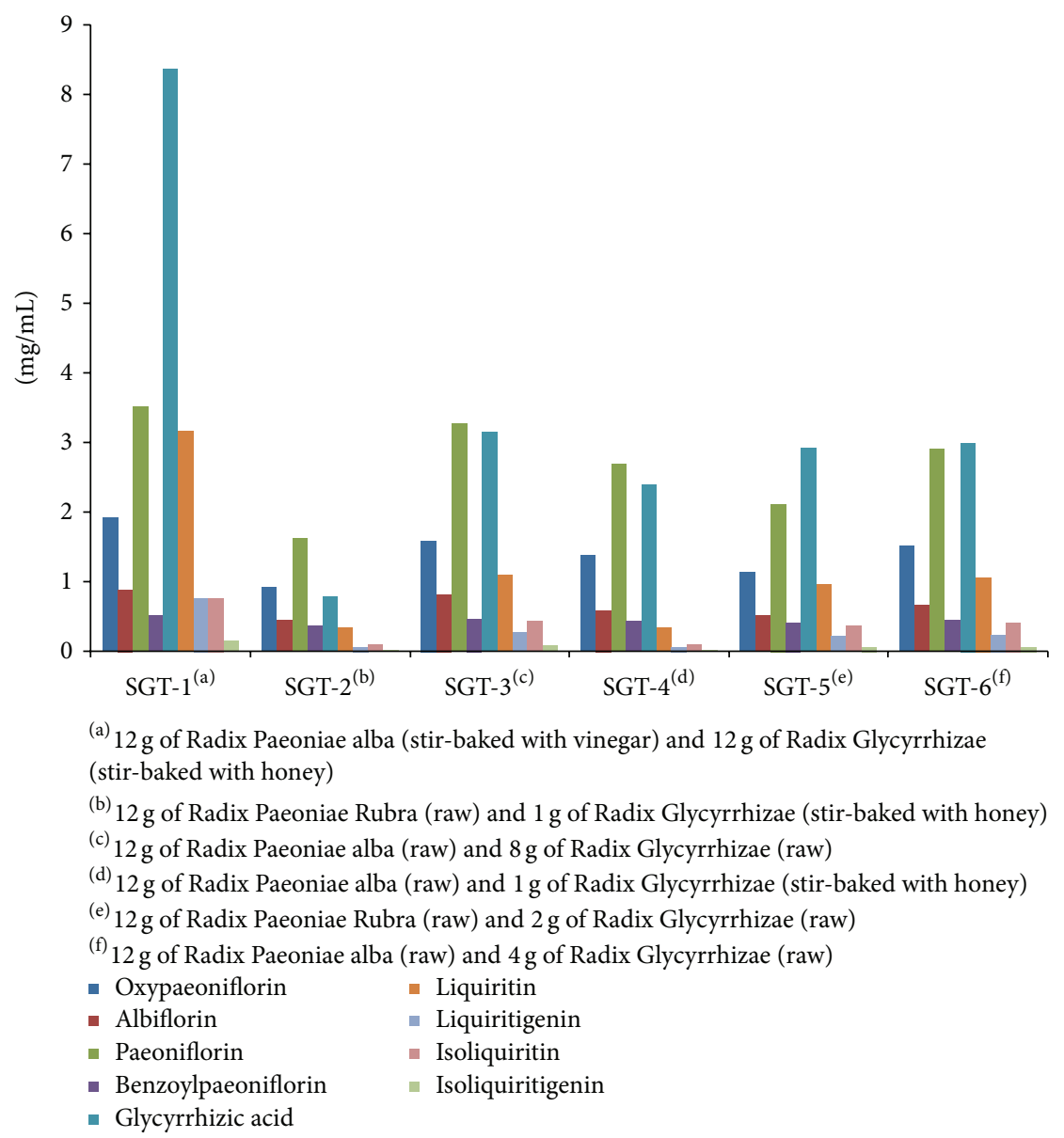

FIGURE 4: The contents of nine compounds in SGT $(\mathrm{mg} / \mathrm{mL})(n=3)$.

and then centrifuged again at $8000 \mathrm{rpm}$ for $20 \mathrm{~min}$ at $4^{\circ} \mathrm{C}$. Then filter the concentrate solution with a $0.45 \mu \mathrm{m}$ filter. For HPLC analysis, the solution with the volume of $1 \mathrm{~mL}$ was dissolved in methanol at a final volume of $10 \mathrm{~mL}$. Before injection, it was filtered through a $0.22 \mu \mathrm{m}$ filter.

2.4. Preparation of Standard Solution. The appropriate amount of oxypaeoniflorin, albiflorin, paeoniflorin, liquiritin, isoliquiritin, liquiritigenin, benzoylpaeoniflorin, isoliquiritigenin, and glycyrrhizic acid were separately weighed and dissolved together in methanol to achieve a standard working solution of nine different concentrations; the concentrations of the nine reference compounds were $42 \mu \mathrm{g} / \mathrm{mL}, 70.2 \mu \mathrm{g} / \mathrm{mL}, 190.8 \mu \mathrm{g} / \mathrm{mL}, 102 \mu \mathrm{g} / \mathrm{mL}, 41.2 \mu \mathrm{g} / \mathrm{mL}$, $44.8 \mu \mathrm{g} / \mathrm{mL}, 47.2 \mu \mathrm{g} / \mathrm{mL}, 4.12 \mu \mathrm{g} / \mathrm{mL}$, and $440 \mu \mathrm{g} / \mathrm{mL}$. The calibration curves were constructed by analyzing the mixed solution, and the series of working solutions within the ranges of $0.84 \sim 8.4 \mu \mathrm{g} / \mathrm{mL}$ for oxypaeoniflorin, $1.4 \sim 14 \mu \mathrm{g} / \mathrm{mL}$ for albiflorin, 3.82 38.2 $\mu \mathrm{g} / \mathrm{mL}$ for paeoniflorin, $2.04 \sim 20.4 \mu \mathrm{g} / \mathrm{mL}$ for liquiritin, $0.08 \sim 0.8 \mu \mathrm{g} / \mathrm{mL}$ for isoliquiritin, $0.9 \sim 9.0 \mu \mathrm{g} / \mathrm{mL}$ for liquiritigenin, $0.94 \sim 9.4 \mu \mathrm{g} / \mathrm{mL}$ for benzoylpaeoniflorin, and $8.8 \sim 88 \mu \mathrm{g} / \mathrm{mL}$ for glycyrrhizic acid, respectively. All solutions were prepared in dark brown calibrated flasks and stored at $4^{\circ} \mathrm{C}$.

2.5. Method Validation. The HPLC method was validated in terms of linearity, precision, stability, repeatability, and recovery. The validation was performed based on the relative peak areas (RPAs). Linear regression analysis was employed to construct calibration curves. The data was expressed as mean \pm standard deviation. And relative standard deviation (RSD) was used to evaluate precision, stability, repeatability, and recovery.

\section{Results}

3.1. Chromatography. The chromatogram of test sample and mixed standard compounds were shown in Figure 3. In chromatograms (a), (b), (c), and (d), the nine peaks marked with 1-9 are oxypaeoniflorin, albiflorin, paeoniflorin, liquiritin, isoliquiritin, liquiritigenin, benzoylpaeoniflorin, isoliquiritigenin, and glycyrrhizic acid. The retention time is $8.514 \mathrm{~min}, 12.195 \mathrm{~min}, 13.599 \mathrm{~min}, 16.525 \mathrm{~min}, 23.957 \mathrm{~min}$, $26.116 \mathrm{~min}, 33.600 \mathrm{~min}, 40.931 \mathrm{~min}$, and $44.705 \mathrm{~min}$, respectively. In chromatograms (a), (c), and (d), the unknown 
TABLE 5: Linear regression equation, linear range, and limit of detections (LODs) for nine standard substances.

\begin{tabular}{|c|c|c|c|c|c|}
\hline $\begin{array}{l}\text { Standard } \\
\text { substance }\end{array}$ & $\begin{array}{c}\text { Linear regression } \\
\text { equation } \\
y=a x+b^{(\mathrm{a})}\end{array}$ & $\begin{array}{c}\text { Correlation } \\
\text { coefficient } \\
\left(r^{2}\right)\end{array}$ & $\begin{array}{l}\text { Linear range } \\
(\mu \mathrm{g} / \mathrm{mL})\end{array}$ & $\begin{array}{c}\text { LOD } \\
(\mathrm{ng} / \mathrm{mL})\end{array}$ & $\begin{array}{c}\text { LOQ } \\
(\mathrm{ng} / \mathrm{mL})\end{array}$ \\
\hline Oxypaeoniflorin & $y=283.4 x+2.053$ & 0.9995 & $0.84 \sim 8.40$ & 3.02 & 9.09 \\
\hline Albiflorin & $y=741.8 x-13.62$ & 0.9997 & $1.40 \sim 14.00$ & 10.32 & 30.86 \\
\hline Paeoniflorin & $y=800.5 x+122.5$ & 0.9995 & $3.82 \sim 38.20$ & 8.34 & 26.02 \\
\hline Benzoylpaeoniflorin & $y=1531 x-51.41$ & 0.9997 & $0.94 \sim 9.40$ & 6.23 & 19.31 \\
\hline Glycyrrhizic acid & $y=376.4 x+22.81$ & 0.9996 & $8.80 \sim 88.00$ & 16.44 & 50.64 \\
\hline Liquiritin & $y=1072 x-73.08$ & 0.9998 & $2.04 \sim 20.40$ & 7.56 & 22.53 \\
\hline Liquiritigenin & $y=4281 x+27.72$ & 0.9995 & $0.90 \sim 9.00$ & 8.24 & 25.54 \\
\hline Isoliquiritin & $y=4025 x+167.9$ & 0.9997 & $0.08 \sim 0.80$ & 20.11 & 63.15 \\
\hline Isoliquiritigenin & $y=23005 x+15.01$ & 0.9998 & $0.82 \sim 8.20$ & 6.89 & 20.60 \\
\hline
\end{tabular}

(a) $y$ and $x$ stand for the peak area and the injection quantity $(\mu \mathrm{g})$ of each standard substance.

TABLE 6: Precision, repeatability, stability, and recovery of nine standard substances.

\begin{tabular}{|c|c|c|c|c|c|}
\hline Standard Substance & $\begin{array}{c}\text { Precision } \\
\text { RSD (\%) } \\
(n=6)\end{array}$ & $\begin{array}{c}\text { Repeatability } \\
\text { RSD (\%) } \\
(n=6)\end{array}$ & $\begin{array}{l}\text { Stability } \\
\text { RSD (\%) } \\
(n=5)\end{array}$ & $\begin{array}{l}\text { Recoveries }(\%)^{(\mathrm{a})} \\
\qquad(\%) \quad(n=9)\end{array}$ & $\begin{array}{l}\text { RSD } \\
(\%)\end{array}$ \\
\hline Oxypaeoniflorin & $1.65 \%$ & $1.00 \%$ & $1.96 \%$ & $99.00 \%$ & $1.28 \%$ \\
\hline Albiflorin & $1.75 \%$ & $1.83 \%$ & $1.19 \%$ & $99.00 \%$ & $1.32 \%$ \\
\hline Paeoniflorin & $0.58 \%$ & $1.84 \%$ & $1.32 \%$ & $99.00 \%$ & $2.12 \%$ \\
\hline Benzoylpaeoniflorin & $1.91 \%$ & $2.00 \%$ & $1.91 \%$ & $100.00 \%$ & $2.40 \%$ \\
\hline Glycyrrhizic acid & $1.64 \%$ & $1.75 \%$ & $1.53 \%$ & $100.00 \%$ & $1.18 \%$ \\
\hline Liquiritin & $1.20 \%$ & $1.56 \%$ & $1.17 \%$ & $101.00 \%$ & $2.33 \%$ \\
\hline Liquiritigenin & $1.02 \%$ & $0.92 \%$ & $1.43 \%$ & $99.00 \%$ & $0.89 \%$ \\
\hline Isoliquiritin & $1.40 \%$ & $1.34 \%$ & $1.43 \%$ & $100.00 \%$ & $0.53 \%$ \\
\hline Isoliquiritigenin & $1.28 \%$ & $0.96 \%$ & $1.29 \%$ & $101.00 \%$ & $2.50 \%$ \\
\hline
\end{tabular}

(a) Recovery $(\%)=100 *$ (amount found - original amount)/amount spiked.

peaks are probably licorice and ketone, licorice alcohol, isoliquiritigenin alcohol, methyl gallate, ethyl gallate, galloyl benzoic acid, and formononetin which was based on published references. Further research will be performed soon in this area in order to provide sufficient evidence for the identification of those unknown peaks.

3.2. Calibration Curves. Linearity was evaluated by analyzing six injection quantities of standard solutions, and then the calibration curves were constructed by plotting the peak areas and the injection quantity of $2 \mu \mathrm{L}, 5 \mu \mathrm{L}, 10 \mu \mathrm{L}, 15 \mu \mathrm{L}$, and $20 \mu \mathrm{L}$ of each compound. Linear regression equation, linear range, limit of detections (LODs), and limit of quantitation (LOQs) for nine standard substances are given in Table 5.

3.3. Precision, Stability, Repeatability, and Recovery. The precision was performed by six replicate determinations of the standard working solution. Stability was evaluated by analyzing the solutions stored at room temperature (about $\left.25^{\circ} \mathrm{C}\right)$ at different time points $(0,4,8,12$, and $24 \mathrm{~h}$ after preparation). The solutions for stability test included mixed solutions of reference standard and SGT sample solutions. Five replicates were performed for the test. These data confirmed that the nine compounds were stable within $24 \mathrm{~h}$ at $25^{\circ} \mathrm{C}$ and their RSD values were between $0.53 \%$ and $2.50 \%$. The repeatability was examined by six replications of a sample. In the recovery test, samples were prepared at three concentration levels in triplicate by spiking known quantities of each of the nine standards into the SGT sample and then extracted and analyzed according to the described procedures. The validation data are shown in Table 6.

3.4. Contents of Nine Compounds in SGT. The HPLC data demonstrated that oxypaeoniflorin, albiflorin, paeoniflorin, benzoylpaeoniflorin, glycyrrhizic acid, liquiritin, isoliquiritin, liquiritigenin, and isoliquiritigenin are present in the extract of SGT. The present study proves that different compatibility of medicines has different contents. The content of nine compounds in SGT is shown in Table 7 and Figure 4.

\section{Discussion}

4.1. Optimization of HPLC Conditions. The combination of acetonitrile and $0.1 \%$ aqueous phosphoric acid $(\mathrm{v} / \mathrm{v})$ proved to be an optimal mobile phase system for the purpose of chromatographic separation compared with acetonitrile 
TABLE 7: The contents of nine compounds in SGT $(\mathrm{mg} / \mathrm{mL})(n=3)$.

\begin{tabular}{|c|c|c|c|c|c|c|}
\hline Compounds & SGT-1 ${ }^{(\mathrm{a})}$ & SGT-2 $2^{(b)}$ & SGT-3 $3^{(\mathrm{c})}$ & SGT- $4^{(\mathrm{d})}$ & SGT- $5^{(\mathrm{e})}$ & SGT-6 ${ }^{(\mathrm{f})}$ \\
\hline Oxypaeoniflorin & 1.92 & 0.92 & 1.59 & 1.38 & 1.14 & 1.52 \\
\hline Albiflorin & 0.90 & 0.45 & 0.82 & 0.59 & 0.52 & 0.67 \\
\hline Paeoniflorin & 3.51 & 1.62 & 3.28 & 2.70 & 2.12 & 2.91 \\
\hline Benzoylpaeoniflorin & 0.53 & 0.37 & 0.47 & 0.43 & 0.41 & 0.45 \\
\hline Glycyrrhizic acid & 8.35 & 0.78 & 3.15 & 2.40 & 2.92 & 3.00 \\
\hline Liquiritin & 3.16 & 0.34 & 1.10 & 0.35 & 0.97 & 1.07 \\
\hline Liquiritigenin & 0.77 & 0.06 & 0.27 & 0.06 & 0.22 & 0.24 \\
\hline Isoliquiritin & 0.77 & 0.10 & 0.44 & 0.10 & 0.36 & 0.42 \\
\hline Isoliquiritigenin & 0.15 & 0.01 & 0.08 & 0.01 & 0.06 & 0.07 \\
\hline Total content of nine compounds & 20.06 & 4.65 & 11.20 & 8.02 & 8.72 & 10.35 \\
\hline
\end{tabular}

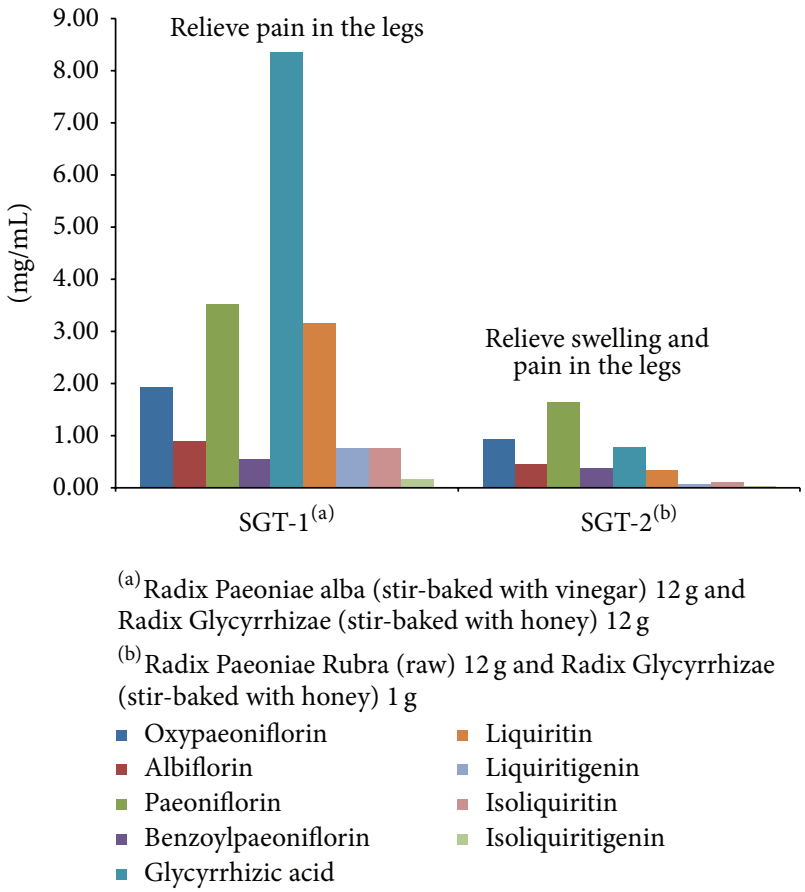

FIGURE 5: Correlation between therapeutical effects and content of SGT-1 and SGT-2.

and $0.02 \%$ aqueous phosphoric acid (v/v), acetonitrile and $0.05 \%$ aqueous phosphoric acid ( $/ \mathrm{v})$. The data showed that separation of peak 2 and peak 3 is not very good in the condition of acetonitrile and $0.02 \%$ aqueous phosphoric acid $(\mathrm{v} / \mathrm{v})$, acetonitrile and $0.05 \%$ aqueous phosphoric acid (v/v). The resolution is about 1.00 . The column temperature was maintained at $30^{\circ} \mathrm{C}$ by comparing with $35^{\circ} \mathrm{C}, 40^{\circ} \mathrm{C}$. The data showed that separation of peak 1 and peak 5 is not very good at $35^{\circ} \mathrm{C}$ and $40^{\circ} \mathrm{C}$. The resolutions were about 1.01 and 1.03 , and the retention times were $7.04 \mathrm{~min}$ and $20.57 \mathrm{~min}$ which were shorter than the normal value. The flow rate of $1 \mathrm{~mL} / \mathrm{min}$ was determined by testing different flow rates which were $0.5 \mathrm{~mL} / \mathrm{min}, 0.8 \mathrm{~mL} / \mathrm{min}$, and $1.5 \mathrm{~mL} / \mathrm{min}$. Final gradient elution method was chosen by testing different gradient elution methods.

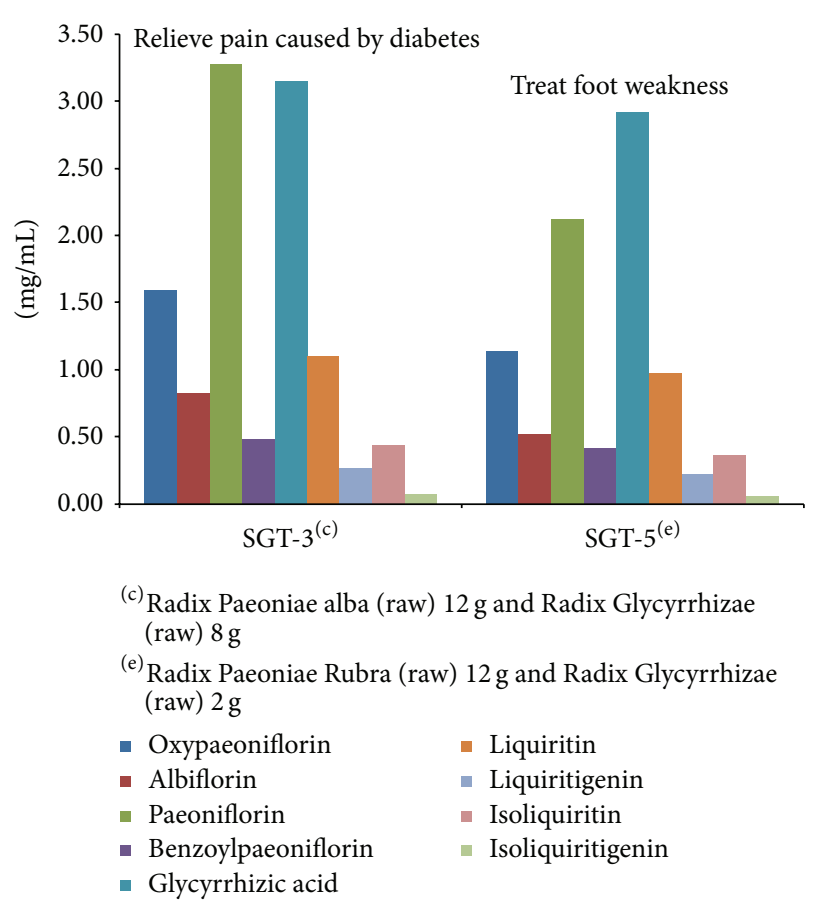

FIGURE 6: Correlation between therapeutical effects and content of SGT-3 and SGT-5.

4.2. Selections of Nine Active Compounds. The theory of Traditional Chinese Medicine holds that the therapeutical effect of Traditional Chinese Medicinal Prescription is due to multicompounds. Oxypaeoniflorin, albiflorin, paeoniflorin, and benzoylpaeoniflorin are the major active compounds of Radix Paeoniae alba. Liquiritin, isoliquiritin, liquiritigenin, isoliquiritigenin, and glycyrrhizic acid are the major active compounds of Radix Glycyrrhizae [30]. The nine compounds all have antispasmodic and analgesic effect. As a result, the nine compounds of SGT should be considered for study.

4.3. Correlation between Content, Dosage, and Compatibility of Medicines. The content of chemical compounds of Traditional Chinese Medicinal Prescription is mainly influenced by the dosage and compatibility of medicines. 


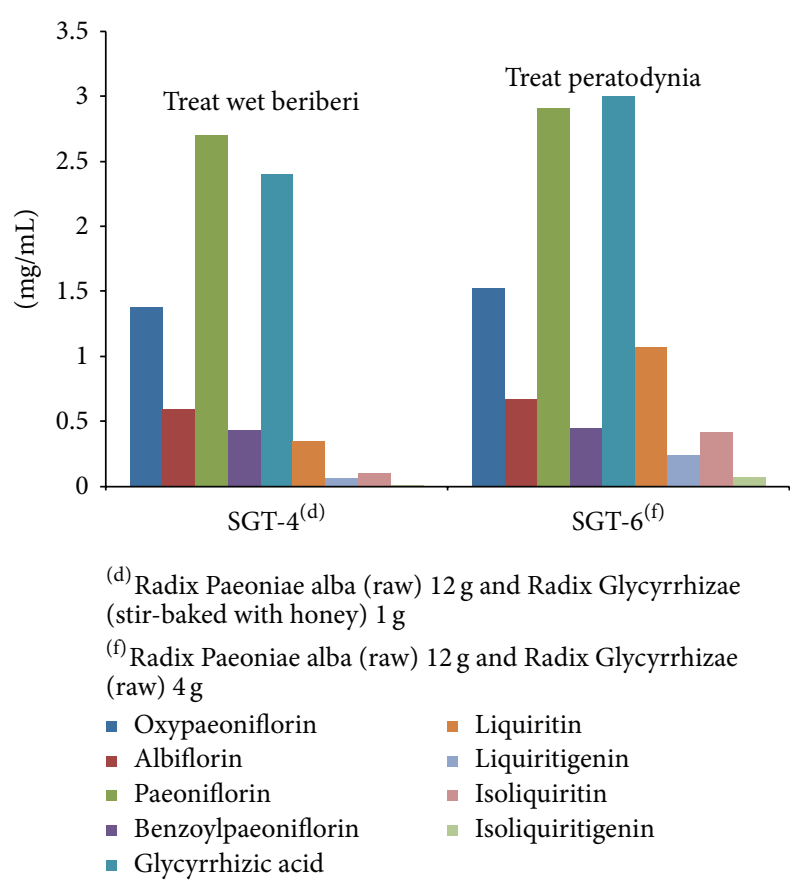

FIGURE 7: Correlation between therapeutical effects and content of SGT-4 and SGT-6.

The dosage of herbs remains constant while the compatibility of herb medicines is changed; different compatibility of herb medicines can lead to different content of compounds. Take SGT-2 and SGT-5 for example: SGT-2 is composed of Radix Paeoniae Rubra (12 g, raw) and Radix Glycyrrhizae (1 g, stir-baked with honey), and SGT-5 is composed of Radix Paeoniae alba (12 g, raw) and Radix Glycyrrhizae (1 g, stirbaked with honey); the two drugs have the same dosage but different herbs. Therefore, the contents of chemical compounds are different. The contents are $(\mathrm{mg} / \mathrm{mL}) 0.92$, $0.45,1.62,0.37,0.78,0.34,0.06,0.10$, and 0.01 and $1.14,0.52$, $2.12,0.41,2.92,0.97,0.22,0.36$, and 0.06 , respectively.

The compatibility of herb medicines remains constant while the dosage level is changed, different dosage of herb medicines can lead to different content of compounds. Take SGT-3 and SGT6 for example: SGT-3 is composed of Radix Paeoniae alba (12 g, raw) and Radix Glycyrrhizae (8 g, raw), and SGT-6 is composed Radix Paeoniae alba (12 g, raw) and Radix Glycyrrhizae (4 g, raw), the two drugs have the same compatibility of medicines but different dosage. Therefore, the content of chemical compounds is also different. The contents are $(\mathrm{mg} / \mathrm{mL}) 1.59,0.82,3.28,0.486,3.15,1.10,0.27$, 0.44 , and 0.08 and $1.52,0.67,2.91,0.45,3.00,1.07,0.24,0.42$, and 0.07 , respectively.

Different dosage and different compatibility of medicines lead to different content. The possible reasons are that the solubility and chemical properties of the compounds would be changed in virtue of the interactions between herb medicines. Some compounds can affect the $\mathrm{PH}$ of the solution and make it acidic or alkaline. Thus, indirectly change the solubility of the other compounds. Sometimes, one compound can react with others and change the chemical properties of the other compounds to form insoluble sediments; these sediments are slowly broken down in the body and gradually play a pharmacodynamics and can make the drug attack slowly, so as to reduce the side effects.

4.4. Correlation between Dosage, Compatibility of Medicines, and Therapeutical Effect. We also found that the therapeutical effect of traditional Chinese Medicinal Prescription is influenced by dosage and compatibility of medicines, Different therapeutical effect due to different dosage and different compatibility of medicines.

One reason is that the contents are different due to different dosage and different compatibility of medicines, which is an important factor in treatment. This conclusion has been already proved and described in the medical classic written by medical expert in ancient china. For example, SGT-1 was used to relieve pain in the legs in Shang-han-lun by Zhongjing Zhang in the Han dynasty and SGT-2 was used to relieve swelling and pain in the legs in Chua-xin-shi-yongfang by Yankui Wu in the Song dynasty (Figure 5); SGT-3 was used to relieve pain caused by diabetes and SGT- 5 was used to treat foot weakness in Zhu-shi-ji-yan-fang by Zhuo Zhu in the Song dynasty (Figure 6); SGT-4 was used to treat wet beriberi in Wei-shi-jia-cang-fang by Xian Wei in the Song dynasty and SGT-6 was used to treat peratodyniain Yi-menba-fa by Hongen Liu in the Qing dynasty (Figure 7).

Another reason is that the compatibility of medicines can play a synergistic effect which can enhance the therapeutical effect. Studies confirmed that glycyrrhizic acid can enhance the therapeutical effect of paeoniflorin on analgesic, antispasmodic, antipyretic, and smooth muscle relaxation.

\section{Conclusions}

The study provides a new HPLC-based method for quality control of the Traditional Chinese Medicinal Prescription SGT. The results of the study showed that SGT mentioned above are different in compatibility of medicines and dosage, and the therapeutic effects of them are different too. The method could be suitable for quality control of SGT with bioactive multicompounds.

\section{Conflict of Interests}

The authors declare no conflict of interests.

\section{Acknowledgments}

The authors acknowledge financial supports from the development and industrialization of Traditional Chinese medicine resources protection and utilization of new product of Traditional Chinese Medicine (no. 10124008-14) and the Sunlight Foundation of Chengdu Pufeide Biotech Co., Ltd. (no. 201403-08-TCM).

\section{References}

[1] P. H. Yen, P. V. Kiem, N. X. Nhiem et al., "A new monoterpene glycoside from the roots of Paeonia lacti-flora increases the 
differentiation of osteoblastic MC3T3-E1 cells," Archives of Pharmacal Research, vol. 30, no. 10, pp. 1179-1185, 2007.

[2] T. Okubo, F. Nagai, T. Seto, K. Satoh, K. Ushiyama, and I. Kano, "The inhibition of phenylhydroquinone-induced oxidative DNA cleavage by constituents of Moutan Cortex and Paeoniae Radix," Biological and Pharmaceutical Bulletin, vol. 23, no. 2, pp. 199-203, 2000.

[3] Y. Q. Zheng, W. Wei, L. Zhu, and J. X. Liu, "Effects and mechanisms of Paeoniflorin, a bioactive glucoside from paeony root, on adjuvant arthritis in rats," Inflammation Research, vol. 56, no. 5, pp. 182-188, 2007.

[4] L. Xiao, Y. Z. Wang, J. Liu, X. T. Luo, Y. Ye, and X. Z. Zhu, "Effects of paeoniflorin on the cerebral infarction, behavioral and cognitive impairments at the chronic stage of transient middle cerebral artery occlusion in rats," Life Sciences, vol. 78, pp. 413-420, 2005.

[5] H. Goto, Y. Shimada, Y. Akechi, K. Kohta, M. Hattori, and K. Terasawa, "Endothelium-dependent vasodilator effect of extract prepared from the roots of Paeonia lactiflora on isolated rat aorta," Planta Medica, vol. 62, no. 5, pp. 436-439, 1996.

[6] K. Tabata, K. Matsumoto, Y. Murakami, and H. Watanabe, "Ameliorative effects of paeoniflorin, a major constituent of peony root, on adenosine $A_{1}$ receptor-mediated impairment of passive avoidance performance and long-term potentiation in the hippocampus," Biological and Pharmaceutical Bulletin, vol. 24, no. 5, pp. 496-500, 2001.

[7] D. M. Chen, L. Xiao, X. Cai, R. Zeng, and X. Z. Zhu, "Involvement of multitargets in paeoniflorin-induced preconditioning," Journal of Pharmacology and Experimental Therapeutics, vol. 319, no. 1, pp. 165-180, 2006.

[8] H. Sato, W. Goto, J. Yamamura et al., "Therapeutic basis of glycyrrhizin on chronic hepatitis B," Antiviral Research, vol. 30, no. 2-3, pp. 171-177, 1996.

[9] T. G. van Rossum, A. G. Vulto, R. A. de Man, J. T. Brouwer, and S. W. Schalm, "Glycyrrhizin as a potential treatment for chronic hepatitis C," Alimentary Pharmacology and Therapeutics, vol. 12, pp. 199-205, 1998.

[10] S. Yasui, K. Fujiwara, A. Tawada, Y. Fukuda, M. Nakano, and O. Yokosuka, "Efficacy of intravenous glycyrrhizin in the early stage of acute onset autoimmune hepatitis," Digestive Diseases and Sciences, vol. 56, no. 2, pp. 3638-3647, 2010.

[11] P. Ferrari, A. Sansonnens, B. Dick, and F. J. Frey, "In vivo $11 \beta$ HSD-2 activity: variability, salt-sensitivity, and effect of licorice," Hypertension, vol. 38, no. 6, pp. 1330-1336, 2001.

[12] M. N. Asl and H. Hosseinzadeh, "Review of pharmacological effects of Glycyrrhiza sp. and its bioactive compounds," Phytotherapy Research, vol. 22, no. 6, pp. 709-724, 2008.

[13] B. Fuhrman, S. Buch, J. Uaya et al., "Licorice extract and its major polyphenol glabridin protect low-density lipoprotein against lipid peroxidation: in vitro and ex vivo studies in humans and in atherosclerotic apolipoprotein E-deficient mice," The American Journal of Clinical Nutrition, vol. 66, pp. 267-275, 1997.

[14] H. Haraguchi, N. Yoshida, H. Ishikawa, Y. Tamura, K. Mizutani, and T. Kinoshita, "Protection of mitochondrial functions against oxidative stresses by isoflavans from Glycyrrhiza glabra," The American Journal of Clinical Nutrition, vol. 52, pp. 219-223, 2000.

[15] A. Friis-Moller, M. Chen, K. Fuursted, S. B. Christensen, and A. Kharazmi, "In vitro antimycobacterial and antilegionella activity of licochalcone a from chinese licorice roots," Planta Medica, vol. 68, no. 5, pp. 416-419, 2002.
[16] G. Yoon, B. Y. Kang, and S. H. Cheon, "Topoisomerase i inhibition and cytotoxicity of licochalcones A and E from Glycyrrhiza inflata," Archives of Pharmacal Research, vol. 30, no. 3, pp. 313-316, 2007.

[17] M. Kimura and I. Kimura, "Blocking effects of a new component, paeoniflorigenone, in paeony root on neuron muscular junctions of frogs and mice," Journal of Pharmacology, vol. 35, p. 61, 1984.

[18] M. Endo, T. Oikawa, S. Nunome, H. Yamada, and T. Hanawa, "Pharmacological analysis for the optimal combination ratio of Shakuyaku and Kanzo in Shakuyakukanzoto," Journal of Traditional Medicines, vol. 24, no. 1, pp. 39-42, 2007.

[19] K. Hashizume, H. Yamagami, Y. Shiomi, and H. Furuya, "The effects of Shakuyaku-Kanzo-To on spasm of the lower limbs accompanying central nervous disorders two case studies," The Japanese Journal of Oriental Medicine, vol. 47, pp. 833-836, 1997.

[20] T. Katsura, "The remarkable effect of Kanzo-to and Shakuyakukanzoto in the treatment of acute abdominal pain," The Japan Society for Oriental Medicine, vol. 46, pp. 293-299, 1995.

[21] F. Hinoshita, Y. Ogura, Y. Suzuki et al., "Effect of orally administered Shao-Yao-Gan-Cao-Tang (Shakuyaku-kanzo-to) on muscle cramps in maintenance hemodialysis patients: a preliminary study," The American Journal of Chinese Medicine, vol. 31, pp. 445-453, 2003.

[22] T. H. Kang, H. Y. Baek, and Y. C. Kim, "Protective effect of Jakyak-Gamcho-tang extract and its constituents against tBHP-induced oxidative damage in HT22 cells," The American Journal of Chinese Medicine, vol. 33, no. 2, pp. 181-189, 2005.

[23] L. Guo, S. Y. Cho, S. S. Kang, S. H. Lee, H. Y. Baek, and Y. S. Kim, "Orthogonal array design for optimizing extraction efficiency of active constituents from Jakyak-Gamcho Decoction, the complex formula of herbal medicines, Paeoniae Radix and Glycyrrhizae Radix," Journal of Ethnopharmacology, vol. 113, no. 2, pp. 306-311, 2007.

[24] T. Katsura, "The remarkable effect of Kanzo-to and Shakuyakukanzoto in the treatment of acute abdominal pain," The Japanese Journal of Oriental Medicine, vol. 46, pp. 293-299, 1995.

[25] S. Arichi and M. Kubo, 1997, vol. 11, pp. 3121-3131.

[26] I. Adamsson, C. Edlund, and C. E. Nord, "Microbial ecology and treatment of Helicobacter pylori infections: review," Journal of Chemotherapy, vol. 12, no. 1, pp. 5-16, 2000.

[27] Q. Y. Wu, Y. Zhou, X. Jin, Y. Guan, M. Xu, and L. F. Liu, "Chromatographic fingerprint and the simultaneous determination of five bioactive components of Geranium carolinianum L. water extract by high performance liquid chromatography," International Journal of Molecular Sciences, vol. 12, no. 12, pp. 8740-8749, 2011.

[28] X. Y. Wu, J. L. Tang, C. Mao, J. Q. Yuan, Y. Qin, and V. C. Chung, "Systematic reviews and meta-analyses of traditional Chinese medicine must search Chinese databases to reduce language bias," Evidence-Based Complementary and Alternative Medicine, vol. 2013, Article ID 812179, 7 pages, 2013.

[29] B. Ma, J. Guo, G. Qi et al., "Epidemiology, quality and reporting characteristics of systematic reviews of traditional Chinese medicine interventions published in Chinese journals," PLoS ONE, vol. 6, no. 5, Article ID e20185, 2011.

[30] P. P. Gan, X. Huang, M. Z. Zhong, M. Sun, F. Qin, and C. H. Zhang, "Simultaneous determination of eight major constituents in the traditional Chinese medicine Shaoyao-GancaoTang by UPLC-PDA," Journal of Medicinal Plants Research, vol. 4, no. 24, pp. 2615-2621, 2010. 


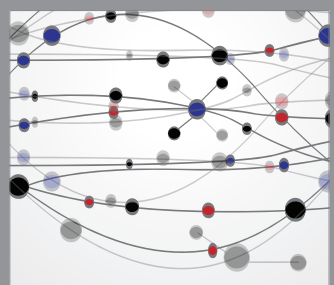

The Scientific World Journal
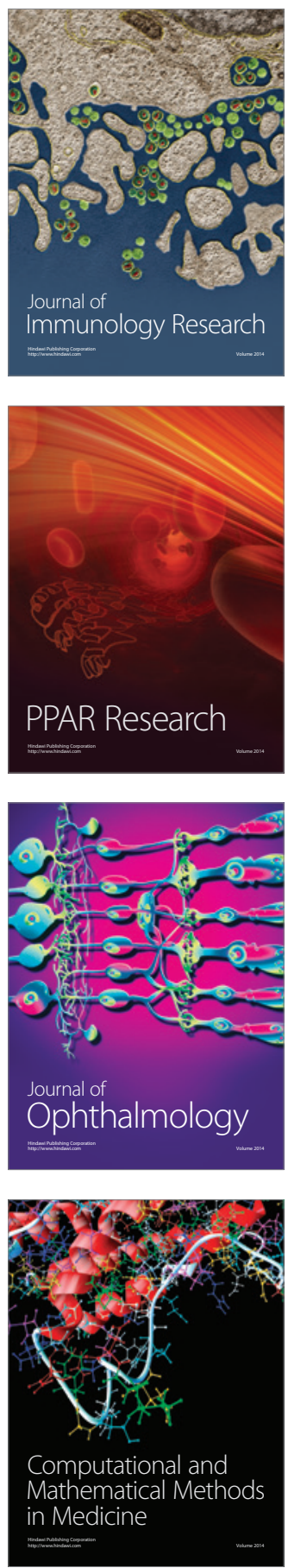

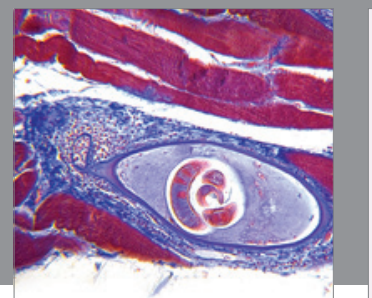

Gastroenterology

Research and Practice
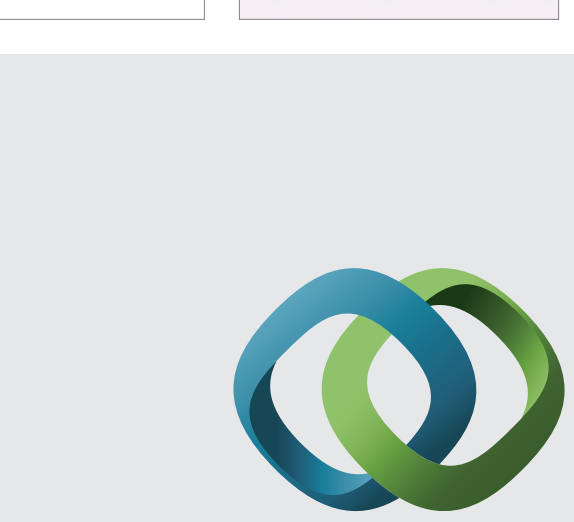

\section{Hindawi}

Submit your manuscripts at

http://www.hindawi.com
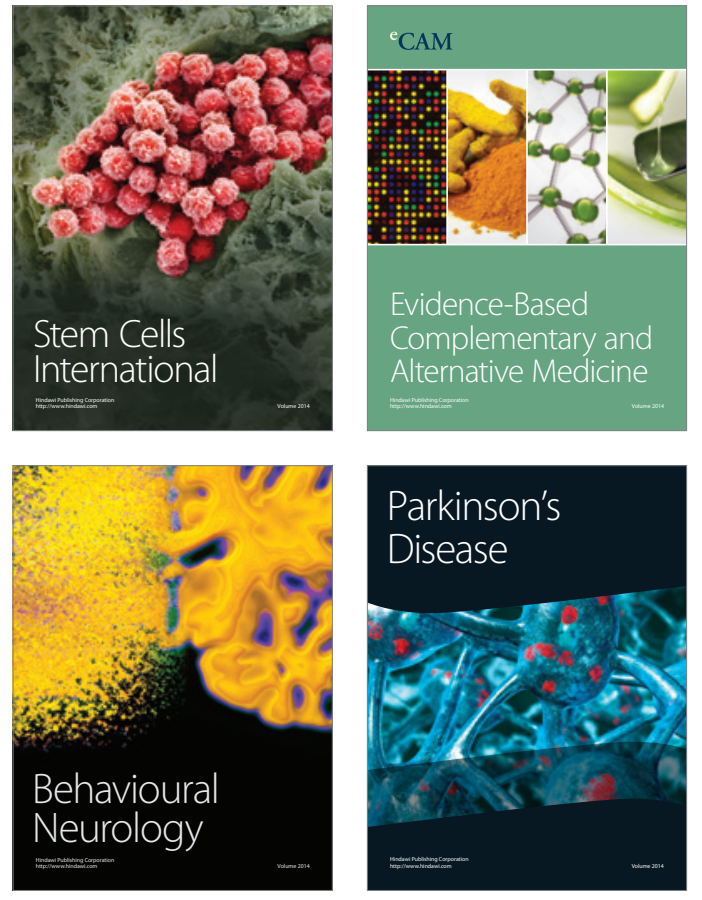
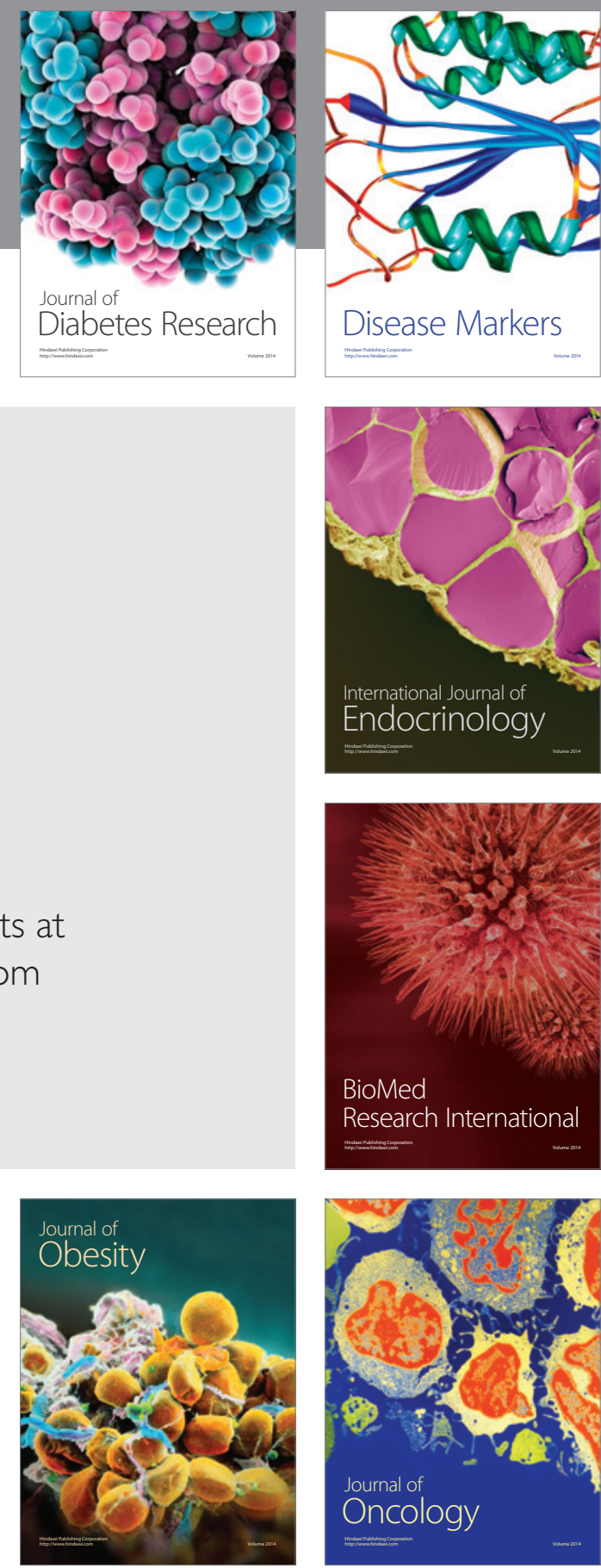

Disease Markers
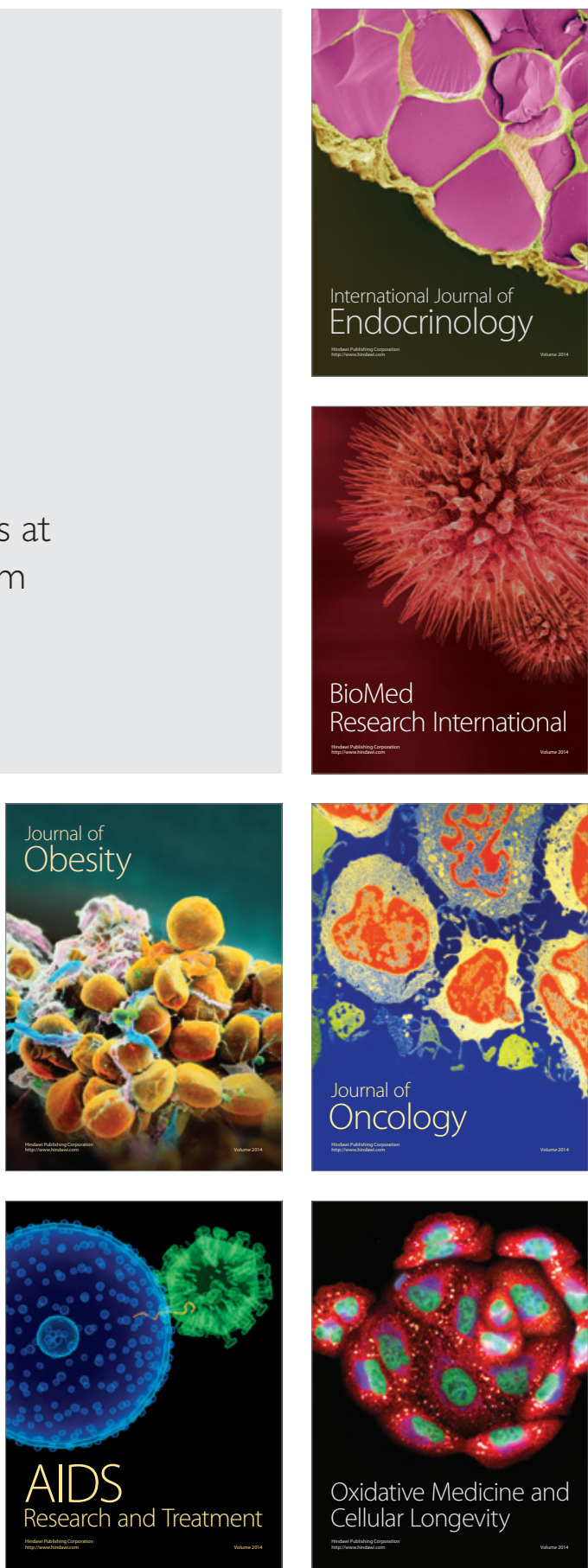